DOI: 10.4274/jerpe.galenos.2021.2021.0053

Case report

\title{
A Possibly Fatal Outcome of Oral Contraceptive Therapy: Estrogen Triggered Hereditary Angioedema Attack in An Adolescent
}

\section{Balkanci et al. Polycystic Ovary Syndrome and F12 Related HAE}

Ugur Berkay Balkanci ${ }^{1}$, Demet Demirkol ${ }^{2,3}$, Gul Yesiltepe Mutlu ${ }^{4}$ Esra Birben ${ }^{5}$, Ozge Soyer ${ }^{5}$, Ozlem Yilmaz ${ }^{6}$, Cansin Sackesen ${ }^{6}$

${ }^{1}$ Koc University, School of Medicine, Istanbul, Turkey

${ }^{2}$ Koc University, School of Medicine, Division of Pediatric Intensive Care, Davutpasa Cad. Istanbul, Turkey

${ }^{3}$ Istanbul University, Istanbul School of Medicine, Division of Pediatric Intensive Care, Istanbul, Turkey; and ${ }^{4} \mathrm{Koc}$ University, School of Medicine, Division of Pediatric Endocrinology, Istanbul, Turkey

${ }^{5}$ Hacettepe University, School of Medicine, Division of Pediatric Allergy, Ankara, Turkey

${ }^{6}$ Koc University, School of Medicine, Division of Pediatric Allergy, Istanbul, Turkey

\begin{abstract}
Hereditary angioedema (HAE) is characterized by recurrent angioedema attacks with no urticaria. This disease has a high mortality due to asphyxia. Level of complement 4 (C4), $\mathrm{C} 1$ esterase inhibitor (C1-INH) level and function, and genetic mutations determine different endotypes of HAE. Clinical presentation and the triggers of vasogenic edema may change according to the endotypes. An adolescent girl with oligomenorrhea, obesity, hirsutism, and acanthosis nigricans was diagnosed with polycystic ovary syndrome (PCOS) and prescribed ethinyl estradiol \& cyproterone acetate containing oral contraceptive (OC). On the $16^{\text {th }}$ day of treatment, she developed angioedema on the face, neck, and chest leading to dyspnea. Adrenaline, antihistamine, and corticosteroid treatments were ineffective. In the family history, the patient's mother and two cousins had angioedema attacks. C1-INH concentrate was administered with a diagnosis of HAE. C4, and C1-INH level and activity were normal. Genetic analysis identified a mutation of Factor XII (F12) gene, and the diagnosis of Factor 12 (F12)related HAE was made. OC treatment discontinued. She has had no additional angioedema attacks in the follow-up period of two years. OC containing estrogen may induce the life-threatening first attack of F12-related HAE even in children. Recurring angioedema attacks in the family should be asked before prescribing estrogen-containing OC pills.
\end{abstract} Keywords: Hereditary Angioedema Type III, Hereditary Angioedema, Angioedema, Factor XII, Polycystic Ovary Syndrome

Cansin Sackesen, Koc University, School of Medicine, Division of Pediatric Allergy, Istanbul, Turkey +905332128787

csackesen@ku.edu.tr; csackesen@yahoo.com

0000-0002-1115-9805

10.03 .2021

15.09.2021

Published: 27 September 2021

\section{Introduction}

Hereditary Angioedema (HAE) is a genetic disorder that presents with an abrupt swelling caused by bradykinin-related vasogenic edema. The mechanism of HAE is non-inflammatory and non-allergic but involves increased production of bradykinin. Until now, five different genes have been identified to cause HAE which are serine protease inhibitor G1 (SERPING1), Factor 12 (F12), plasminogen (PLG), angiopoietin (ANGPT1), and kininogen (KNG1) (1-3). Different types of HAE consist of low $\mathrm{C} 1$ inhibitor (C1-INH) activity caused by low production or loss of function of C1-INH due to SERPING1 gene mutations, or normal level and activity of C1-INH (HAEnCI) due to mutations of F12, PLG, ANGPT1, and KNG1 genes. HAEnCI presentation is like the other forms of HAE which are characterized by recurring attacks of angioedema without urticaria that can be fatal due to laryngeal swellings. In this form of the disease, the most common defective plasma protein is F12 $(3,4)$. Factor XII is a critical molecule where pathways of coagulation, complement activation, contact reaction, and fibrinolysis meet. The promoter region of the $F 12$ gene carries an estrogen-responsive element and there are multiple case reports that associate high levels of estrogen and the HAE attacks (5-7).

This manuscript describes a patient presenting with her first angioedema attack after initiation of oral contraceptive treatment for the polycystic ovary syndrome (PCOS) that was subsequently diagnosed with HAEnCI with F12 mutation.

Case

A thirteen-year-old girl presented to the emergency department with dyspnea and swelling in her upper body. Her medical history was notable for PCOS which was diagnosed 2 weeks ago in another pediatric endocrinology clinic. She was reported to have irregular menstruation and hirsutism and diagnosed with PCOS with high androgen levels (Table 1) and polycystic ovary morphology in pelvic ultrasound, and ethinyl estradiol \& cyproterone acetate containing OC pill was initiated. On the $16^{\text {th }}$ day of treatment, she developed periorbital swelling which spread over the face, the neck, and the upper body in matters of hours (Figure 1). With the initial diagnosis of anaphylaxis, epinephrine, antihistamines, and steroids were administered but her swellings were unresponsive. Her family history was positive for recurring angioedema attacks for her mother and two cousins. Therefore, she was given $500 \mathrm{IU}$ of $\mathrm{C} 1$ esterase inhibitor concentrate with a pre-diagnosis of HAE. She was internalized to the Koc University Pediatric Intensive Care Unit due to the possible risk of laryngeal edema. During her stay in pediatric intensive care, 2 more doses of $500 \mathrm{IU}$ of $\mathrm{C} 1$ esterase inhibitor concentrate were given. The swellings started 
receding in 12 hours and they have completely waned within 48 hours. The physical examination was also remarkable for obesity with a BMI of $29.7 \mathrm{~kg} / \mathrm{m}^{2}\left(>99^{\text {th }}\right.$ percentile, $+2.51 \mathrm{SDS}$, acanthosis nigricans on the neck, and severe hirsutism with a Ferriman-Gallway score of 25.

Her laboratory workup showed complement 4 levels of $31 \mathrm{mg} / \mathrm{dL}$ (normal range:10-40 mg/dL) and normal plasma levels $(0.31 \mathrm{~g} / \mathrm{L}$, normal range:0.21-0.39 $\mathrm{g} / \mathrm{L})$ and activity of $\mathrm{C} 1$ esterase inhibitor $(107.7 \%$, normal range:70-130\%) which indicated a diagnosis of HAEnCI. Genetic analysis of F12 gene revealed a heterozygous mutation at the ninth exon, C $>\mathrm{A}$ variant which result in p.Thr328Lys.

OC pill was discontinued, life-style interventions were recommended. No attack occurred during her follow-up for 20 months.

Discussion

We report a female adolescent who had got the diagnosis of PCOS in another clinic and presented with angioedema attack after OC medication initiated. The final diagnosis of HAEnCI was made with genetic analysis of $F 12$ gene showing a heterozygous mutation. F12 related HAEnCI is an autosomal dominant disease that shows incomplete penetrance. In most cases, the first symptoms appear before the third decade. Published series of HAE patients report a clear female predominance (8). Also, women are more likely to be symptomatic than men. Hormonal factors play an important role in the worsening of the condition in women. There are differences in the overall frequency of angioedema symptoms according to different female life stages of childhood, adolescence, menstruation, pregnancy, and menopause. It has also been reported that administration of estrogen, not progestin, in women with HAE may lead to the emergence or worsening of angioedema symptoms (7). A case series conducted on 61 women with F12 related HAEnCI showed that $95 \%$ of the women presented with at least one angioedema attack during periods of high estrogen exposure (OC pill, hormone replacement therapy, or pregnancy) (4). The estrogen as a trigger for HAE attacks could not be explained by a single mechanism. However, limited literature indicates that the main culprit could be the estrogen-responsive element on the 5' flank of the Factor XII gene $(5,6)$. Another possible effect of estrogens in the pathogenesis of HAE is that estrogen-containing medications can decrease angiotensin-converting enzyme, which is also a protein responsible for degradation of bradykinin. Once angiotensinconverting enzyme activity decreased, accumulation of bradykinin could occur (7). Although some authors proposed that PCOS might have a protective role regarding HAE attacks due to increased levels of androgen and more stable levels of estradiol in PCOS patients (7), compelling evidence showing the protective effect of hyperandrogenism is lacking. The frequent co-occurrence of PCOS and HAE may suggest a link between neuroendocrine and immune system consisting of the presence of a pathology related with hypothalamic-pituitary dysregulation and an immunological disorder (6). However, the relationship between these two disorders needs to be explained.

The present case also reminds us of the challenges associated with the diagnosis of PCOS in adolescence. Diagnostic criteria for PCOS in adolescence remain controversial, because the diaghostic pathological features including irregular menses 2 years beyond menarche, cystic acne, and polycystic ovarian morphology used in adult women may be normal pubertal physiological events (9). Our case had a history of oligomenorrhea and hirsutismus. She had presented to another pediatric endocrinology clinic with these chief complaints. The peivic ultrasound which was performed in that center showed PCO morphology and laboratory tests revealed a high level of 1,4 androstenedione but a normal testosterone level. Although serum free testosterone level was unavailable, the low level of sex hormone binding globulin suggests it might be elevated. After being diagnosed with PCOS she was started on OC therapy. No pharmacological treatment has been approved so far by FDA/EMA for use in adolescents with PCOS; however, some pharmacological interventions including OC have been frequently used to manage PCOS symptoms (9). Our treatment approach in our patient was discontinuing the estrogencontaining OC pill and life-style interventions (calorie restricted diet, exercise and behavioral treatment) to provide weight loss. There are some reports showing the efficacy of progestin-only OC in decreasing the attack incidence in HAE (10-11), however the use of them in adolescence is debatable. Another pillar of treatment in HAE is patient education. Patients should be advised to avoid estrogen-containing products and cooperate with their doctors when planning to become pregnant in the future.

To the best of our knowledge this case is the first pediatric case of hereditary angioedema due to factor 12 mutation that is induced by estradiol containing OC in the literature. The individuals with HAE-related mutations may not have any attack until encountering a trigger like estrogen-containing drugs. Thus, we highlight the importance of obtaining a thorough family history regarding any HAE attack before initiation of OC as it can be lifesaving.

Conflict of interest disclosures: All authors declare that they have no affiliation with or involvement in any organization or entity with any financial or non-financial interest in the subject matter discussed in this manuscript.

References

1. Banday AZ, Kaur A, Jindal AK, Rawat A, Singh S. An update on the genetics and pathogenesis of hereditary angioedema. Genes Dis 2020; 7:75-83.

2. Bork K, Wulff K, Rossmann H, et al. Hereditary angioedema cosegregating with a novel kininogen 1 gene mutation changing the N-terminal cleavage site of bradykinin. Allergy 2019; 74:2479-2481.

3. Zuraw B.L. Hereditary angioedema with normal C1 inhibitor: Four types and counting. J Allergy Clin Immunol 2018; 141:884-885.

4. Bork K, Wulff K, Witzke G, Hardt J. Hereditary angioedema with normal C1-INH with versus without specific F12 gene mutations. Allergy 2015; 70:1004-1012.

5. Farsetti A, Misiti S, Citarella F, et al. Molecular basis of estrogen regulation of Hageman factor XII gene expression. Endocrinology 1995; 136:5076-5083.

6. Perricone R, Pasetto N, De Carolis C, et al. Cystic ovaries in women affected with hereditary angioedema. Clin Exp Immunol 1992; 90:401-404.

7. Iahn-Aun M, Aun MV, Motta AA, et al. The Complex Interaction Between Polycystic Ovary Syndrome and Hereditary Angioedema: Case Reports and Review of the Literature. Obstet Gynecol Surv 2017; 72:417-424. 
8. Bork K, Meng G, Staubach P, et al. Hereditary angioedema: new findings concerning symptoms, affected organs, and course. Am J Med. 2006;119:267-274.)

9. Ibáñez L, Oberfield SE, Witchel S, Auchus RJ, Chang RJ, Codner E, Dabadghao P, Darendeliler F, Elbarbary NS, Gambineri A, Garcia Rudaz C, Hoeger KM, López-Bermejo A, Ong K, Peña AS, Reinehr T, Santoro N, Tena-Sempere M, Tao R, Yildiz BO, Alkhayyat H, Deeb A, Joel D, Horikawa R, de Zegher F, Lee PA. An International Consortium Update: Pathophysiology, Diagnosis, and Treatment of Polycystic Ovarian Syndrome in Adolescence. Horm Res Paediatr. 2017;88(6):371-395.

10. Bork K, Wulff K, Witzke G, Hardt J. Treatment for hereditary angioedema with normal C1-INH and specific mutations in the F12 gene (HAE-FXII). Allergy 2017; 72:320-324.

11. Saule C, Boccon-Gibod I, Fain O, et al. Benefits of progestin contraception in non-allergic angioedema. Clin Exp Allergy 2013; 43:475-482.

Table 1. Laboratory tests showing serum hormone levels

\begin{tabular}{|l|l|l|}
\hline & Value & Normal range \\
\hline 1,4-Androstenedion $(\mathrm{ng} / \mathrm{mL})$ & 2.38 & $0.24-1.73$ \\
\hline Testosterone $(\mathrm{ng} / \mathrm{mL})$ & 0.24 & $0.24-1.67$ \\
\hline Sex hormone binding globulin $(\mathrm{nmol} / \mathrm{L})$ & 10.6 & $11-120$ \\
\hline 17-alpha hydroxyprogesterone $(\mathrm{ng} / \mathrm{dL})$ & 107.0 & $\frac{13-185}{60-100}$ \\
\hline Fasting blood glucose $(\mathrm{mg} / \mathrm{dL})$ & 91.0 & $\frac{60}{2.6-25}$ \\
\hline Fasting insulin $(\mu \mathrm{U} / \mathrm{mL})$ & 66.8 & \\
\hline
\end{tabular}




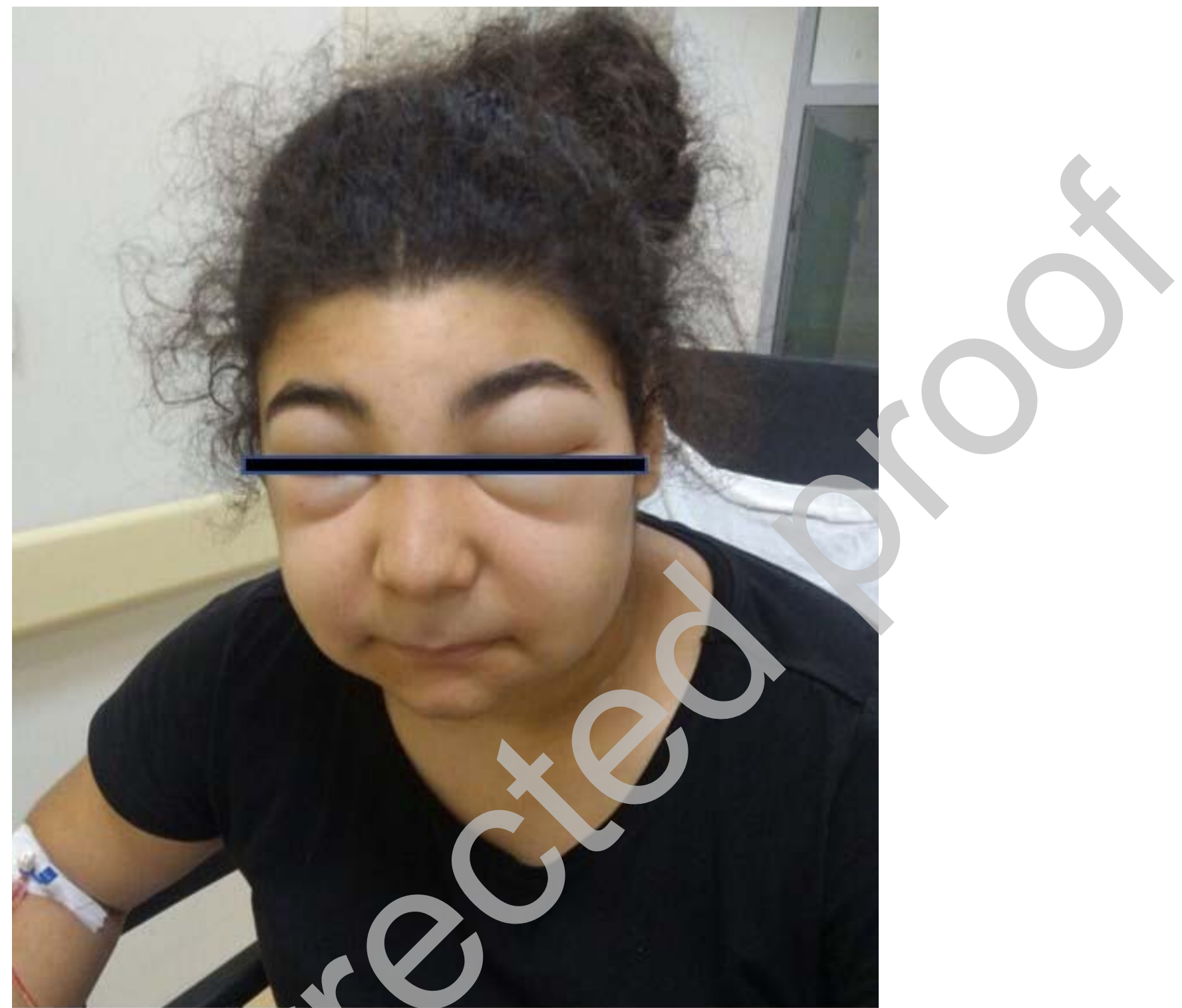

Figure 1. The face of the patient at admission

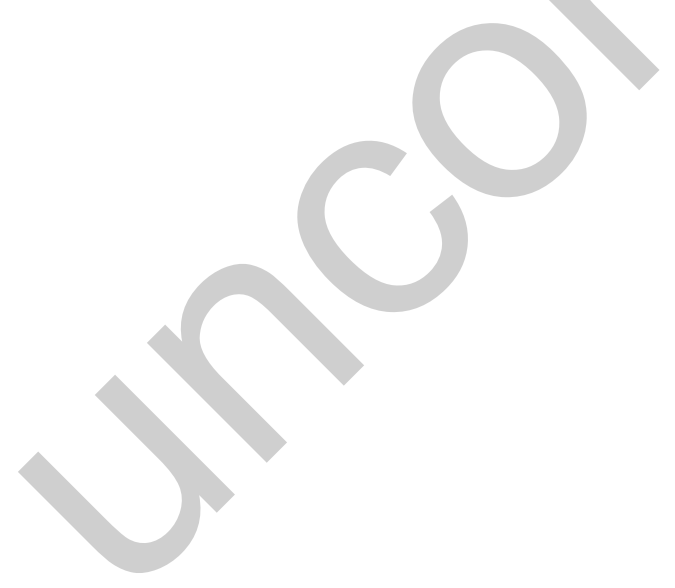

